\title{
ON REAL ZEROS OF RANDOM POLYNOMIALS WITH HYPERBOLIC ELEMENTS
}

\author{
K. FARAHMAND \\ Department of Mathematics \\ University of Ulster \\ Jordanstown \\ Co. Antrim BT37 0QH, U.K.

\section{JAHANGIRI} \\ Department of Mathematics \\ Kent State University \\ 14111 Claridon Troy Road \\ Burton, OH 44021-9500, U.S.A.
}

(Received May 1, 1996 and in revised form December 30, 1996)

\begin{abstract}
This paper provides the asymptotic estimate for the expected number of real zeros of a random hyperbolic polynomial $g_{1} \cosh x+2 g_{2} \cosh 2 x+\ldots+n g_{n} \cosh n x$ where $g_{j},(j=$ $1,2, \ldots, n)$ are independent normally distributed random variables with mean zero and variance one. It is shown that for sufficiently large $n$ this asymptotic value is $(1 / \pi) \log n$.
\end{abstract}

KEY WORDS AND PHRASES: Number of real zeros, Kac-Rice formula, random algebraic polynomial, random trigonometric polynomial.

1991 AMS SUBJECT CLASSIFICATION CODES: Primary 60G99; Secondary 42BXX.

\section{INTRODUCTION}

Let $(\Omega, \mathcal{A}, \operatorname{Pr})$ be a fixed probability space and $\left\{g_{j}(\omega)\right\}_{j=1}^{n}$ be a sequence of independent identically distributed random variables defined on $\Omega$, each normally distributed with mean zero and variance one. Denote by $M_{n}(\alpha, \beta)$ the number of real zeros of $P_{n}(x)$ in the interval $(\alpha, \beta)$, where,

$$
P_{n}(x) \equiv P_{n}(x, \omega)=\sum_{j=1}^{n} j g_{j}(\omega) \cosh j x,
$$

and by $E M_{n}(\alpha, \beta)$ its expected value. A similar hyperbolic polynomial to (1.1) has been studied in an unpublished work of Das [4] reported by Bharucha-Reid and Sambandham [1, page 110]. As oscillatory behavior of hyperbolic functions is well known it would be interesting to know how far this oscillatory behavior is transferred to the random hyperbolic polynomial. Since $E M_{n}(-\infty, \infty)$ could serve as the expected number of maxima and minima of a random hyperbolic polynomial, its value together with the expected number of real zeros and $K$-level crossings gives a better picture of such oscillations. This expected number of maxima and minima for other type of random polynomials, such as the random algebraic polynomial

$$
F(x) \equiv F_{n}(x, \omega)=\sum_{\jmath=1}^{n} g_{\jmath}(\omega) x^{\jmath},
$$


and the random trigonometric polynomial

$$
T(x) \equiv T_{n}(x, \omega)=\sum_{j=1}^{n} g_{j}(\omega) \cos j x,
$$

is obtained by Das [3] and [2], respectively. It is shown that in the algebraic case the expected number of maxima and minima is asymptotic to $\{(\sqrt{3}+1) / \pi\} \log n$, that is about 1.366 times $(2 / \pi) \log n$, the expected number of real zeros, see also Wilkins [9]. Therefore in the algebraic case there are about $(0.366) \log n$ oscillations which do not occur between two axis crossings, while for the trigonometric polynomial the expected number of maxima and minima in the interval $(0,2 \pi)$ is $2 \sqrt{3} n / \sqrt{5}$, which is about 1.342 times $2 n / \sqrt{3}$, the expected number of real zeros, see also Farahmand [6] and Wilkins [10]. Hence in the latter case, asymptotically, there are about 0.197 oscillations which do not occur between two axis crossings. It is, therefore, of special interest to have equivalent results for the hyperbolic case. We prove the following:

THEOREM 1 The expected number of real zeros of $P_{n}(x)$ as $n \rightarrow \infty$, satisfies

$$
E M_{n}(-\infty, \infty) \sim(1 / \pi) \log n
$$

From the proof of the theorem we will see that the above expected number arises from interval $(-1,1)$ and in $(-\infty,-1) \cup(1, \infty)$ there is no significant number of zeros. This is also the case for the expected number of real zeros of $\sum_{j=1}^{n} g_{j}(\omega) \cosh j x$. The latter expected number is given in [4], or [5], as $(1 / \pi) \log n$. Therefore, our theorem leads to the conjecture that for the hyperbolic case the number of oscillations are the same as the number of zero crossings. That is, for the hyperbolic case almost all the oscillations occur between two zero crossings. Indeed, unlike the algebraic case, these oscillations are all in the interval $(-1,1)$ and outside this interval the polynomial does not oscillate significantly.

\section{PRIMARY ANALYSIS}

The Kac-Rice formula, [7] and [8], gives the expected number of real zeros of $P_{n}(x)$ in the interval $(\alpha, \beta)$ as

where

$$
E N(\alpha, \beta)=\int_{\alpha}^{\beta}\left(\Delta / \pi A^{2}\right) d x
$$

$$
\begin{aligned}
A^{2} & =\operatorname{var}\left\{P_{n}(x)\right\}=\sum_{j=1}^{n} j^{2} \cosh ^{2} j x, \\
B^{2} & =\operatorname{var}\left\{P_{n}^{\prime}(x)\right\}=\sum_{j=1}^{n} j^{4} \sinh ^{2} j x, \\
C & =\operatorname{cov}\left\{P_{n}(x), P_{n}^{\prime}(x)\right\}=\sum_{j=1}^{n} j^{3} \sinh j x \cosh j x
\end{aligned}
$$

and

$$
\Delta^{2}=A^{2} B^{2}-C^{2}
$$

Since for each zero of $P_{n}(x)$ in the positive $x$-axis there corresponds a zero of $P_{n}(x)$ in the negative $x$-axis, it is sufficient to restrict ourselves to $(0, \infty)$. Successive differentiation of both sides of

$$
\sum_{j=1}^{n} \cosh 2 j x=\cosh n x \sinh (n+1) x / \sinh x-1,
$$

which is easy to prove, together with (2.2)-(2.4) and a little algebra yield,

$$
A^{2}=n(n+1)(2 n+1) / 12+\sinh (2 n+1) x\{n(n+1)
$$




$$
\begin{aligned}
& \left.+\cosh ^{2} x / 2 \sinh ^{2} x\right\} / 4 \sinh x-(2 n+1) \cosh x \cosh (2 n+1) x / 8 \sinh ^{2} x, \\
B^{2}= & -n(n+1)(2 n+1)\left(3 n^{2}+3 n-1\right) / 60+\left\{n\left(n^{3}+2 n^{2}-1\right)\right. \\
& \left.+3 \cosh ^{4} x / 2 \sinh ^{4} x\right\} \sinh (2 n+1) x / 4 \sinh x-\left\{2\left(2 n^{3}+3 n^{2}-n-1\right)\right. \\
& \left.+(6 n+3) \cosh ^{2} x / \sinh ^{2} x\right\} \cosh x \cosh (2 n+1) x / 8 \sinh ^{2} x \\
& +\left\{\left(3 n^{2}+3 n-1\right) \cosh ^{2} x \sinh (2 n+1) x / 4 \sinh ^{3} x\right.
\end{aligned}
$$

and

$$
\begin{aligned}
C= & (16 \sinh x)^{-1}\left[\left\{\left(4 n^{3}+6 n^{2}-1\right)\right.\right. \\
& \left.+3(2 n+1) \cosh ^{2} x / \sinh ^{2} x\right\} \cosh (2 n+1) x \\
& \left.-\left\{\left(6 n^{2}+6 n-1\right)+3 \cosh ^{2} x / \sinh ^{2} x\right\} \cosh x \sinh (2 n+1) x / \sinh x\right]
\end{aligned}
$$

In order to be able to carry out the integration required in (2.1) we seek to obtain the dominant terms in (2.6)-(2.8). To this end we first let $x \in(\epsilon, 1)$ where $\epsilon=(\log n)^{1 / 2} / n$. Since in this interval, for all sufficiently large $n, 2 n+1>p \operatorname{coth} x \tanh (2 n+1) x$, for $p=1,2, \ldots, 5$ then $f_{n, p}(x)=\sinh (2 n+1) x / \sinh ^{p} x$ is an increasing function of $x$. Therefore, since $\sinh x<4 x$ in $(0,1)$ for all sufficiently large $n$,

$$
f_{n, p} \geq(n / 4)^{p}(\log n)^{-p / 2} \exp \left\{2(\log n)^{1 / 2}\right\}
$$

Hence, those terms in (2.6)-(2.8) that are appearing in the form of $f_{n, p}$ for the largest value of $p$ are dominant. Hence from (2.5) and (2.6)-(2.8) we can easily obtain,

$$
\begin{aligned}
A^{2} & =\left\{n^{2} \sinh (2 n+1) x / 4 \sinh x\right\}\left\{1+O(\log n)^{-1 / 2}\right\} \\
B^{2} & =\left\{n^{4} \sinh (2 n+1) x / 4 \sinh x\right\}\left\{1+O(\log n)^{-1 / 2}\right\} \\
C & =\left\{n^{3} \cosh (2 n+1) x / 4 \sinh x\right\}\left\{1+O(\log n)^{-1 / 2}\right\}
\end{aligned}
$$

In order to evaluate $\Delta^{2}=A^{2} B^{2}-C^{2}$ the dominant terms obtained in (2.9)-(2.11) somehow cancel out. Therefore we need to use (2.6)-(2.8). Some algebra, together with the same application of the upper limit of $f_{n, p}$ mentioned above yields,

$$
\Delta^{2}=\left\{n^{4} \sinh (2 n+1) x \cosh (2 n+1) x / 64 \sinh ^{4} x\right\}\left\{1+O(\log n)^{-1 / 2}\right\} .
$$

These estimations are sufficient to enable us to prove the theorem.

\section{PROOF OF THE THEOREM}

We first avoid getting too close to zero by confining ourselves to the interval $(\epsilon, 1)$ where as above $\epsilon=(\log n)^{1 / 2} / n$. We can, therefore, use the estimates obtained in (2.9)-(2.12). Thus using (2.1), since for all $x \in(\epsilon, 1)$ and $n$ sufficiently large $\operatorname{coth}(2 n+1) x=1+o(\log n)^{-1 / 2}$, some algebra yields,

$$
\begin{aligned}
E M_{n}(\epsilon, 1) & =(1 / 2 \pi) \int_{\epsilon}^{1} \operatorname{csch} x d x\left\{1+O(\log n)^{-1 / 2}\right\} \\
& =(1 / 2 \pi) \log n\left\{1+O(\log n)^{-1 / 2}\right\} .
\end{aligned}
$$

Now we show that the expected number of maximum and minimum in the interval $(0, \epsilon)$ is small. To this end from (2.1) and the definition of $A^{2}$ and $B^{2}$ in (2.2) and (2.3) we can write,

$$
\begin{aligned}
E M_{n}(0, \epsilon) & <\int_{0}^{\epsilon}(B / \pi A) d x \\
& =\int_{0}^{\epsilon}\left\{\sum_{j=1}^{n} j^{4} \sinh ^{2} j x / \pi^{2} \sum_{j=1}^{n} j^{2} \cosh ^{2} j x\right\}^{1 / 2} d x \\
& <n \epsilon / \pi=O(\log n)^{1 / 2} .
\end{aligned}
$$


Therefore (3.1) and (3.2) gives $(1 / 2 \pi) \log n$ as the asymptotic value for $E M_{n}(0,1)$ and so.

$$
E M_{n}(-1,1) \sim(1 / \pi) \log n .
$$

Now it only remains to consider the case of $x \in(-\infty,-1) \cup(1, \infty)$. As we mentioned above it suffices to confine ourselves to the interval $(1, \infty)$. In this interval also the first leading terms of $A^{2}, B^{2}$ and $C$ which appeared in (2.6)-(2.8) in the evaluation of $\Delta^{2}$, cancel out. Therefore we need to use the following estimates, valid for sufficiently large $n$,

$$
\begin{aligned}
& A^{2} \sim n\{(n+1) \sinh (2 n+1) x-\operatorname{coth} x \operatorname{coth}(2 n+1) x\} / 4 \sinh x, \\
& B^{2} \sim n^{3}\{(2 n+4-\operatorname{coth} x) \sinh (2 n+1) x-3 \operatorname{coth} x \cosh (2 n+1) x\} / 8 \sinh x, \\
& C \sim n^{2}\{(2 n+3) \cosh (2 n+1) x-3 \operatorname{coth} x \sinh (2 n+1) x\} / 8 \sinh x .
\end{aligned}
$$

Therefore from (2.5) and (3.4)-(3.6) for all sufficiently large $n$ we obtain

$$
\Delta^{2} \sim n^{5} \cosh x \sin (2 n+1) x\{\cosh (2 n+1) x-\sinh (2 n+1) x\} / 32 \sinh ^{3} x .
$$

Finally from (2.1), (3.7) and (3.4) we have,

$$
\begin{aligned}
E M_{n}(1, \infty) \sim & (\sqrt{n} / \pi \sqrt{2}) \int_{1}^{\infty} \operatorname{coth}^{1 / 2} x \sinh ^{-1 / 2}(2 n+1) x\{\cosh (2 n+1) x \\
& -\sinh (2 n+1) x\}^{1 / 2} d x \\
< & (\sqrt{2 n} / \pi) \int_{1}^{\infty} \operatorname{coth}^{1 / 2} x \exp (-n x) d x \\
< & o\left(n^{-1 / 2}\right) .
\end{aligned}
$$

Since (3.8) can also serve as an upper limit for $E M_{n}(-\infty,-1)$ from (3.3) we have proof of the theorem.

ACKNOWLEDGMENT: The authors wish to thank the referee for useful comments on the earlier version of this paper.

\section{References}

[1] A.T. Bharucha-Reid and M. Sambandham. Random Polynomials. Academic Press, N.Y., 1986.

[2] M. Das. The average number of real zeros of a random trigonometric polynomial. Proc. Camb. Phil. Soc., 64:721-729, 1968.

[3] M. Das. The average number of maxima of a random algebraic curve. Proc. Camb. Phil. Soc., 65:741-753, 1969.

[4] M. Das. On the real zeros of a random polynomial with hyperbolic elements. Unpublished Ph.D Dissertation, India, 1971.

[5] K. Farahmand. Level crossings of a random polynomial with hyperbolic elements. Proc. Amer. Math. Soc., In Press.

[6] K. Farahmand. The level crossings of random polynomials. Appl. Math. Lett., 9:19-25, 1996.

[7] M. Kac. On the average number of real roots of a random algebraic equation. Bull.Amer.Math.Soc., 49:314-320, 1943.

[8] S.O. Rice. Mathematical theory of random noise. Bell. System Tech. J., 25:46-156, 1945.

[9] J.E. Wilkins. An asymptotic expansion for the expected number of real zeros of a random polynomial. Proc. Amer. Math. Soc., 103:1249-1258, 1988.

[10] J.E. Wilkins. Mean number of real zeros of a random trigonometric polynomial. Proc. Amer. Math. Soc., 111:851-863, 1991. 


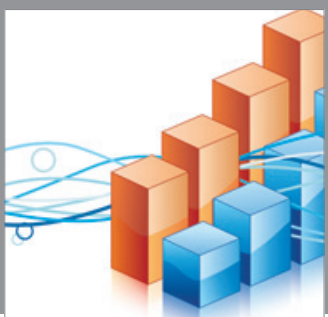

Advances in

Operations Research

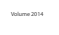

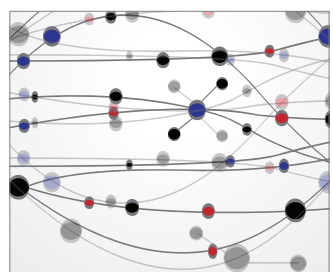

\section{The Scientific} World Journal
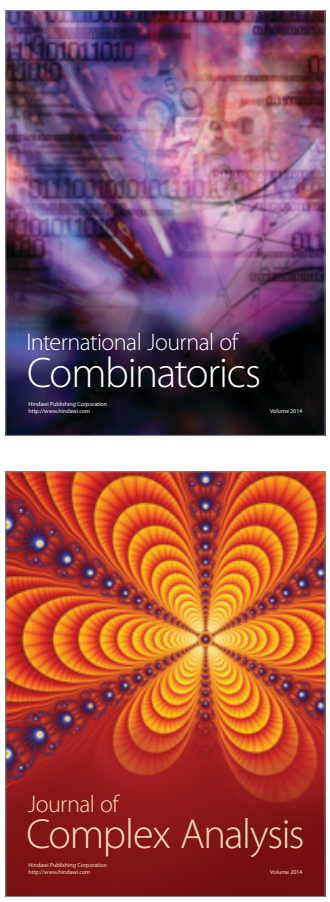

International Journal of

Mathematics and

Mathematical

Sciences
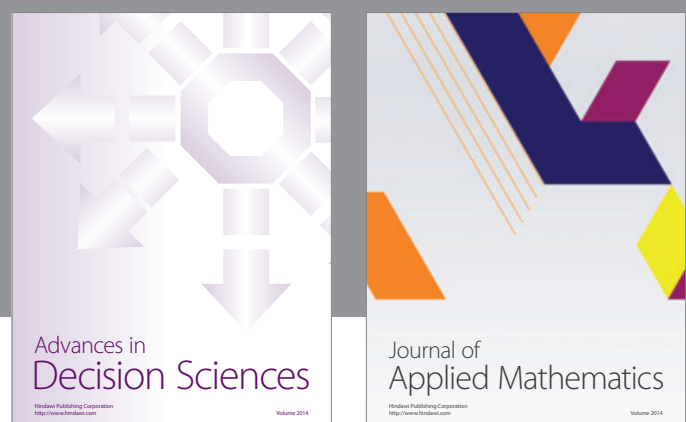

Journal of

Applied Mathematics
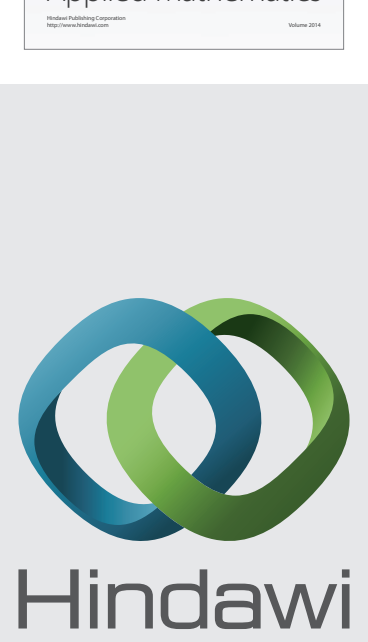

Submit your manuscripts at http://www.hindawi.com
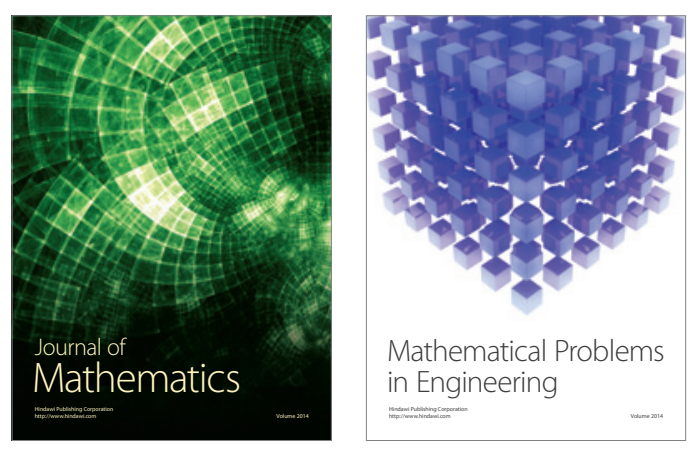

Mathematical Problems in Engineering
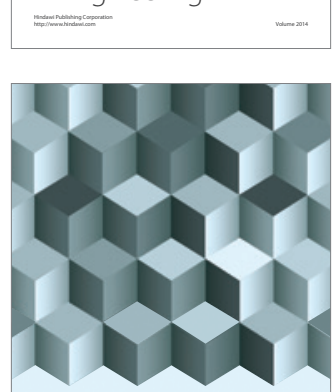

Journal of

Function Spaces
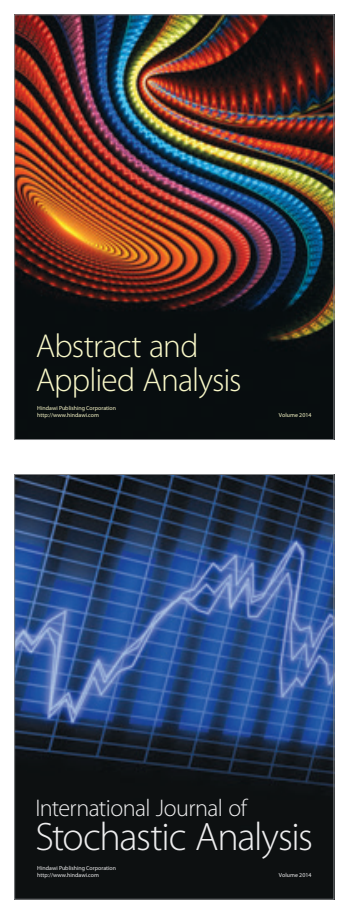

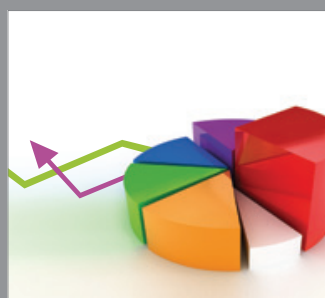

ournal of

Probability and Statistics

Promensencen
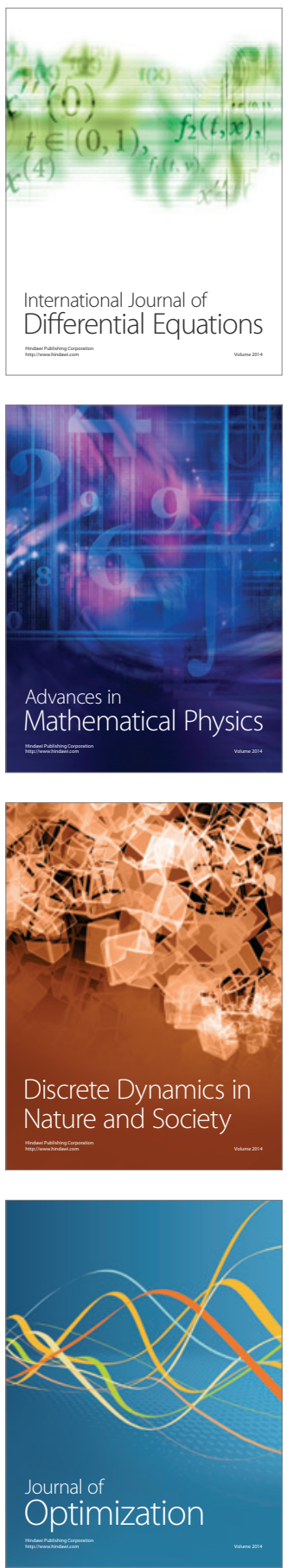